\title{
Defective heat shock factor 1 inhibits the growth of fibrosarcoma derived from simian virus 40/T antigen-transformed MEF cells
}

\author{
QIYING JIANG, ZHI ZHANG, SHULIAN LI, ZHAOYANG WANG, \\ YUANFANG MA and YANZHONG HU
}

\author{
Henan Key Laboratory of Engineering Antibody Medicine, Henan International Union Laboratory of \\ Antibody Medicine and Chaperone, Henan University School of Medicine, Kaifeng, Henan 475004, P.R. China
}

Received June 23, 2014; Accepted August 17, 2015

DOI: $10.3892 / \mathrm{mmr} .2015 .4300$

\begin{abstract}
Heat shock factor 1 (Hsf1) serves an important role in regulating the proliferation of human tumor cell lines in vitro and tissue specific tumorigenesis in certain mouse models. However, its role in viral-oncogenesis remains to be fully elucidated. In the current study, the role of Hsf 1 in fibroblastoma derived from simian virus $40 / \mathrm{T}$ antigen (SV40/TAG)-transformed mouse embryonic fibroblast (MEF) cell lines was investigated. Knockout of Hsf 1 inhibited MEF cell proliferation in vitro and fibroblastoma growth and metastasis to the lungs in vivo in nude mice. Knockout of Hsf1 increased the protein expression levels of p53 and phosphorylated retinoblastoma protein $(\mathrm{pRb})$, however reduced the expression of heat shock protein 25 (Hsp25) in addition to the expression of the angiogenesis markers vascular endothelial growth factor, cluster of differentiation 34 and factor VIII related antigen. Furthermore, immunoprecipitation indicated that knockout of Hsf1 inhibited the association between SV40/TAG and p53 or $\mathrm{pRb}$. These data suggest that Hsf1 is involved in the regulation of SV40/TAG-derived fibroblastoma growth and metastasis by modulating the association between SV40/TAG and tumor suppressor p53 and pRb. The current study provides further evidence that Hsf1 may be a novel therapeutic target in the treatment of cancer.
\end{abstract}

\section{Introduction}

Heat shock factor 1 (Hsf1) is the predominant regulator of the heat shock response and has been demonstrated to be

Correspondence to: Dr Yuanfang Ma or Dr Yanzhong $\mathrm{Hu}$, Henan Key Laboratory of Engineering Antibody Medicine, Henan International Union Laboratory of Antibody Medicine and Chaperone, Henan University School of Medicine, 300 Jin-Ming Road, Kaifeng, Henan 475004, P.R. China

E-mail: mayf@henu.edu.cn

E-mail: yhu@henu.edu.cn

Key words: heat shock factor 1, simian virus 40/T antigen, p53, tumorigenesis, phosphorylated retinoblastoma protein associated with certain tissue specific tumorigenesis (1). Hsf 1 protein is upregulated in malignant tumor tissues of the liver, esophagus, prostate, lymphatic system, lungs and breasts $(2,3)$. Inhibition of Hsf1 protein expression suppresses the growth of certain tumor cell lines and upregulates their sensitivity to chemotherapeutic agents in vitro $(4,5)$. In animal models, Hsf1 knockdown inhibits 7,12-dimethylbenz(a) anthracene-induced skin cancer (6), p53 mutation-induced lymphoma, n-nitrosodiethylamine-induced hepatocellular carcinoma (HCC) (2) and epidermal growth factor receptor II (ErbB2)-associated breast cancer (7). Hsf1 has been associated with multiple pathways involved in tumorigenesis. For example, Hsf1 participates in regulating tumor cell protein synthesis, glucose and lipid metabolism, p53 protein stability (8), chromosome stability, the signal transduction of ErbB2 (7) and expression of certain non-heat shock proteins $(6,9)$. These data support the role of Hsf1 as a potential novel target in cancer therapy.

Numerous previous studies have indicated that the Hsf1-mediated heat shock response is critical in modulating cell transformation resulting from viral oncoproteins, which are important for tissue specific tumorigenesis, for example human papillomavirus 16 (HPV16) early genes E6-E7 for cervical carcinoma, adenovirus early region $1 \mathrm{~A}$ (E1A) for adenoma of the prostate and nasal carcinoma and hepatitis $\mathrm{B}$ virus-hepatitis B protein (HBV-HBx) for HCC. For example, $\mathrm{HBx}$ activates Hsf1, which is involved in the upregulation of HBx-induced hepatocyte proliferation (10). Deletion of Hsf1 is able to inhibit E1A-induced mouse embryonic fibroblast (MEF) cell proliferation in vitro (11). These examples demonstrate certain pathways involving Hsf1, however further studies are required to fully elucidate the association between Hsf1 and viral oncoproteins in tumorigenesis.

Simian virus 40 (SV40) is a double stranded DNA virus that is normally expressed in monkey kidney and human brain tumor and malignant mesothelioma tissue (12). Infection with SV40 leads to animal tumors (12), however it is unclear whether SV40 has a similar effect in humans. The proteins that SV40 encodes, the large T-antigen (TAG) and small t-antigen (TAG), are strong viral carcinogens and have been widely used to immortalize normal cells in in vitro tumorigenesis studies (13). TAG binds to protein phosphatase 2A (PP2A) and blocks the tumor suppressor activity of PP2A $(14,15)$. TAG however, is 
able to transform host cells by binding to and inactivating the tumor suppressors p53 and phosphorylated retinoblastoma protein $(\mathrm{pRb})(16)$. In addition to its association with tumor suppressors, SV40/TAG is able to induce the expression of molecular chaperones such as heat shock protein 70 (Hsp70) and binding immunoglobulin protein, which in turn promote the cell transformation activity of SV40/TAG $(16,17)$. Hsf1 is a unique transcription factor of Hsp70. This suggests that the Hsf1-mediated heat shock response may be important for SV40/TAG-induced cell transformation.

The aim of the current study was to investigate the roles of Hsf1 in the tumorigenesis of SV40/TAG-transformed MEF cells, by comparing the effects of Hsf1 knockout MEF cells (MEF/Hsf1-/-), MEF/Hsf1-/- expressing mouse Hsf1 cDNA (MEf/mHsf1) and wild type (wt) MEF cells. The tumor formation and metastatic capabilities of SV40/TAG-transformed MEF cells was investigated in athymic nude mice. The protein expression levels of the angiogenesis markers; cluster of differentiation 34 (CD34), vascular endothelial growth factor (VEGF) and factor VIII related antigen (FVIII/Rag) were investigated immunohistochemically in the resulting tumor tissues. Using western blotting, the expression levels of p53 and $\mathrm{pRb}$ were measured, in addition to a range of heat shock proteins. Coimmunoprecipitation was used to investigate proteins which associate with SV40/TAG.

\section{Materials and methods}

Cell lines and plasmids. MEF/wt and MEF/Hsf1-/- cells were generated from E12.5 embryos from a C57B16/V129 background (donated by Dr Xianzhong Xiao from the Central South University School of Medicine, Changsha, China). The cells were transiently transfected with pcDNA-SV40/TAG (Addgene, Cambridge MA 02139 USA) and immortalized by passaging the cells for a maximum of 30 generations. To generate the MEF/mHsf1 cell line, the retroviral packaging cell line HEK293-ampho cells (American Type Culture Collection, Mansassas, VA, USA) were transiently transfected with the recombinant retrovirus vector $4 \mathrm{~g}$ pWZL-Blasticitin-mFlag-Hsf1. Following a 24-h transfection, the supernatants were collected by centrifugation at $960 \mathrm{x} \mathrm{g}$ for $10 \mathrm{~min}$ and mixed with $2 \mu \mathrm{g} / \mathrm{ml}$ polybrene (Sigma-Aldrich, St. Louis, MO, USA) and used to infect the MEF/Hsf1-/- cell lines, generating the $\mathrm{MEF} / \mathrm{mHsf} 1$ cell line.

Athymic nude mice subcutaneous engraftment assay. Thirty male Balb c-nu/nu specific pathogen free athymic nude mice, (4 weeks old; body weight $16 \mathrm{~g}$ ) were purchased from the Experimental Animal Research Institute of the Chinese Academy of Medical Sciences (Beijing, China). Mice were housed in a sterile room and sacrificed by placing in a sealed box containing $\mathrm{CO}_{2}$. The protocol approved by the Animal Core Facility of the Experimental Animal Research Institute of the Chinese Academy of Medical Sciences (no. HUSOM 2015-036). Each mouse was anesthetized by injection of $0.1 \mathrm{ml} / 10 \mathrm{~g}$ body weight FFm-mix (Fentanyl citrate, Fluanisone and Midazolam). For the athymic nude mice engraftment assay, $5 \times 10^{5}$ cells were subcutaneously injected into the craniodorsal area of the right leg. Following engraftment, the mice were continuously fed for a maximum of 50 days. The time taken for tumor formation was recorded, and the tumor volume was measured using the formula $a(b) 2 / 2$ (where a represents the longest tumor diameter and $\mathrm{b}$ represents the shortest tumor diameter) (18).

MTT and colony formation assays. For the MTT assay, $10^{3}$ cells were seeded in 96-well plates and cultured for 24,48 , 72 and 96 h. The cells were incubated with Dulbecco's modified Eagle's medium (DMEM; Thermo Fisher Scientific, Grand Island, NY, USA) containing $20 \mu \mathrm{M}$ MTT (Life Technologies, Grand Island, NY, USA) for $4 \mathrm{~h}$ and terminated by adding $0.1 \% \mathrm{NP}-40(0.1 \mathrm{ml}) /$ isopropanol lysis buffer $(100 \mathrm{ml})$ for $10 \mathrm{~min}$. The absorbance was measured at a wavelength of $590 \mathrm{~nm}$ using Tecan Infinite F500 (Tecan Männedorf, Switzerland). For the colony formation assay, $10^{3}$ cells were seeded into the 6-well plates and cultured for 7 days. The cells were washed three times with phosphate-buffered saline (PBS) and stained with $1 \%$ crystal violet (Sigma-Aldrich) for $20 \mathrm{~min}$. The number of colonies was then counted manually.

Cell cycle analysis. The cells were synchronized by serum starvation and seeded into 6 -well plates at a concentration of $5 \times 10^{5}$, then were cultured for $24 \mathrm{~h}$ in complete media (DMEM with $1 \mathrm{X}$ penicillin-streptomycin, $10 \mathrm{mM}$ glutamine and $10 \%$ fetal bovine serum; all from Thermo Fisher Scientific). The cells were then trypsinized (Invitrogen Life Technologies, Grand Island, NY, USA) and fixed in $70 \%$ precooled alcohol overnight at $4{ }^{\circ} \mathrm{C}$. The cells were washed in PBS twice to remove the ethanol and resuspended in $500 \mu \mathrm{l}$ pyridine iodide solution (containing $50 \mu \mathrm{g} / \mathrm{ml}$ RNase-A; Invitrogen Life Technologies) in the dark for $30 \mathrm{~min}$ at room temperature. The cell cycle was then measured using flow cytometry (FACSCalibur; BD Biosciences, San Jose, CA, USA).

Immunohistochemistry. Tumor tissue was fixed in $4 \%$ paraformaldehyde/PBS for 1 week, embedded in paraffin and sliced into $4 \mu \mathrm{m}$ tissue slices by a microtome (Leica RM 2235 , Wetzlar, Germany). Paraffin-embedded tumor tissues were deparaffinized and quenched in $3 \% \mathrm{H}_{2} \mathrm{O}_{2}$ (Sigma-Aldrich) to remove the endogenous peroxidase activity. Following antigen retrieval in 0.01 M sodium folic acid buffer (Sigma-Aldrich), the slides were blocked in $10 \%$ normal rabbit serum (Santa Cruz Biotechnology, Inc., Dallas TX, USA) for $30 \mathrm{~min}$. Subsequently, the slides were incubated with the following primary antibodies: Mouse monoclonal anti-VEGF (Santa Cruz Biotechnology, Inc.; cat no. SC-7269; dilution 1:100), mouse monoclonal anti-CD34 antibody (Santa Cruz Biotechnology, Inc., cat. no. SC-7324; dilution 1:100), mouse monoclonal anti-FVIII/Rag antibody (America Diognostica USA, cat. no. ESvWF-10; dilution 1:100), overnight at $4^{\circ} \mathrm{C}$. Slides were then incubated with HRP-conjugated anti-rabbit IgG and HRP-conjugated anti-mouse IgG secondary antibody (Santa Cruz Biotechnology, Inc., dilution 1:200). The signal was developed with diaminobenzidine (Sigma-Aldrich) and the slides were counterstained with hematoxylin and eosin (Sigma-Aldrich). The expression of VEGF, CD34 and FVIII/Rag were quantified as previously published (19). Student's t-test was used for statistical analysis. For measurement of the VEGF expression in the tumor tissues, the brown positive signals were measured by Image Pro-Plus software. 
A

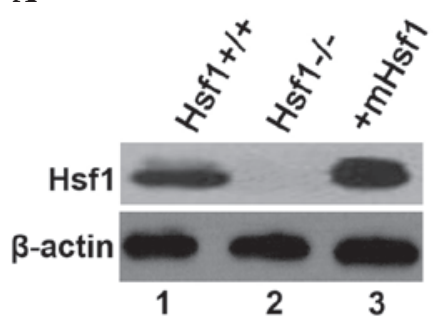

B

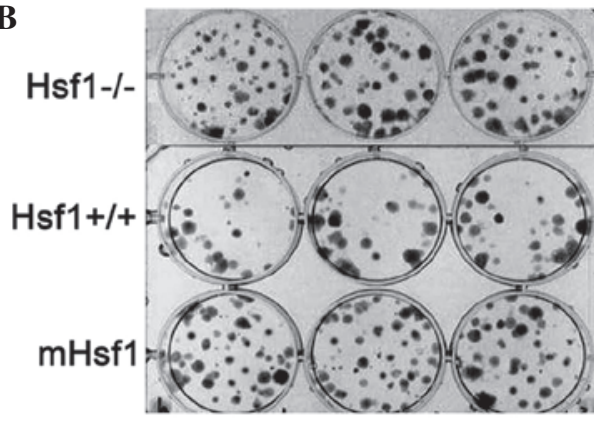

C

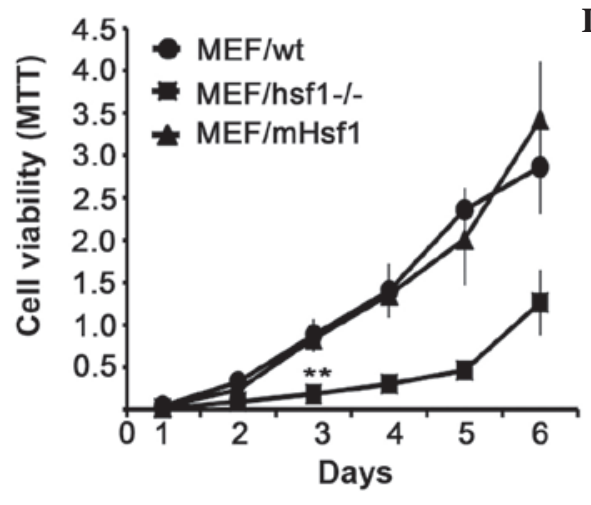

D

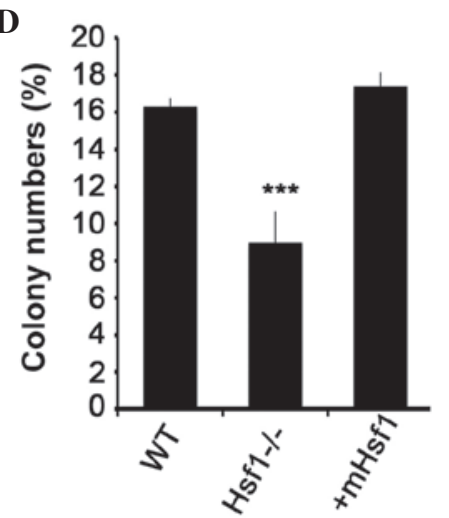

E

Figure 1. Hsf1 knockout inhibits MEF cell proliferation. (A) Expression of Hsf1 proteins in the SV40/TAG-transformed MEF cell lines: Lane 1, MEF/wt; lane 2, MEF/Hsf1-/-; and lane 3, MEF/mHsf1. (B) Clone formation of the three MEF cell lines in flat cloning assay. (C) The growth viability of the three MEF cell lines in an MTT assay. (D) The quantification of colony-forming efficiency of the three MEF cell lines by flat cloning assay. (E) The effects of Hsf1 on the cell cycle of the three MEF cells. ${ }^{*} \mathrm{P}<0.05,{ }^{* *} \mathrm{P}<0.01,{ }^{* * * *} \mathrm{P}<0.001, \mathrm{MEF} / \mathrm{Hsf} 1-/-$ cells vs. MEF/wt and MEF/mHsf1 cells. Hsf1, heat shock factor 1 ; MEF, mouse embryonic fibroblast; SV40/TAG, simian virus 40/T antigen; wt, wild type; mHsf1, Hsf1 null MEF cells expressing mouse Hsf1.

The integral optical density of 10 fields was measured and averaged. The immunohistochemistry staining signal density of CD34 and FVIII/Rag were measured in 10 randomly selected fields. The average of 10 views was used to represent the new vessel grown in the tumor tissues.

Western blotting and coimmunoprecipitation. The cells were lysed in NP-lysis buffer (50 mM Tris-HCl, $\mathrm{pH} 7.4 ; 150 \mathrm{mM} \mathrm{NaCl}$, $1 \%$ NP-40 and $1 \mathrm{X}$ cocktail protease inhibitor (Sigma-Aldrich). The protein concentration was measured using a bicinchoninic acid assay kit (Thermo Fisher Scientific). Equal amounts of protein were separated by $10 \%$ SDS-PAGE (Invitrogen Life Technologies) and transferred onto nitrocellulose membranes (Invitrogen Life Technologies). The membranes were blocked with 5\% non-fat dried milk/Tris-buffered saline-Tween 20 and incubated with the primary antibodies at $4^{\circ} \mathrm{C}$ overnight. The antibodies used were as follows: Rabbit polyclonal anti-Hsf1 antibody (Cell signaling; cat. no. 4356; working dilution 1:1,000), rabbit anti-Hsp25 antibody (Sigma-Aldrich; cat. no. H0148; working dilution 1:2,000), mouse anti-Hsp70 antibody (Enzo Life Sciences, Inc., Farmingdale, NY, USA; cat. no. ADI-SPA-810; working dilution 1:1,000), mouse anti-heat shock cognate protein 70 (Hsc70) antibody (Enzo Life Sciences, Inc.; cat. no. ADI-SPA-820; working dilution 1:1,000), mouse anti-Hsp90 $\alpha$ antibody (Enzo Life Sciences, Inc.; cat. no. ADI-SPA-835; working dilution 1:1,000). Membranes were subsequently incubated with horseradish peroxidase-conjugated secondary antibodies for $1 \mathrm{~h}$ at room temperature. The membranes were developed using enhanced chemiluminescence and exposed to X-ray film (Thermo
Fisher Scientific). Protein quantification was performed with Quantity One 4.6 software (Bio-Rad Laboratories, Inc., Hercules, CA, USA). For immunoprecipitation, the assay was performed as previously published (20). Briefly, $1 \mathrm{mg}$ protein was pre-cleaned with $30 \mu \mathrm{l}$ protein-A agarose beads and then incubated with $2 \mu \mathrm{g}$ antibody at $4^{\circ} \mathrm{C}$ overnight. Subsequently, the sample was incubated with protein $\mathrm{A}$ agarose beads (Invitrogen Life Technologies) for $2 \mathrm{~h}$. The immunoprecipitated protein complexes were subjected to immunoblotting with rabbit anti-SV40/TAG (cat. no. V-300), rabbit anti-p53 (cat. no. sc-6243) and pRB (cat. no. sc-7905) antibodies (Santa Cruz Biotechnology, Inc.).

Statistical analysis. Statistical analysis was performed using SPSS software, version 13.0 (SPSS, Inc., Chicago, IL, USA). Student's t-test was used for paired data that were normally distributed. Comparisons among values of more than two groups were performed using analysis of variance. $\mathrm{P}<0.05$ was considered to indicate a statistically significant difference.

\section{Results}

Hsf1 promotes SV40-immortalized MEF cell proliferation by regulating the cell cycle at the $G_{1}$ and $S$ phases. In order to determine the role of Hsf1 in the cell transformation induced by SV40/TAG, three genotypes of SV40/TAG transformed MEF cell lines were established: MEF/wt, MEF/Hsf1-/- and $\mathrm{MEF} / \mathrm{mHsf1}$ cells (MEF/Hsf1-/- cells expressing mouse Hsf1 cDNA). The expression of Hsf 1 in these three cell lines was investigated by immunoblotting. As presented in Fig. 1A, Hsf1 


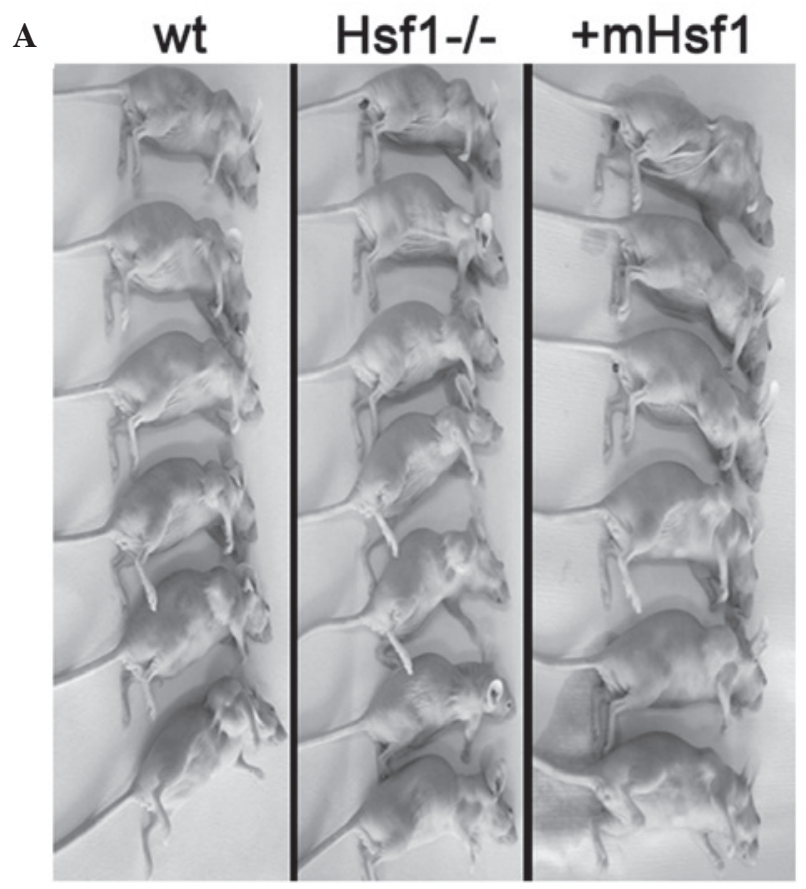

B
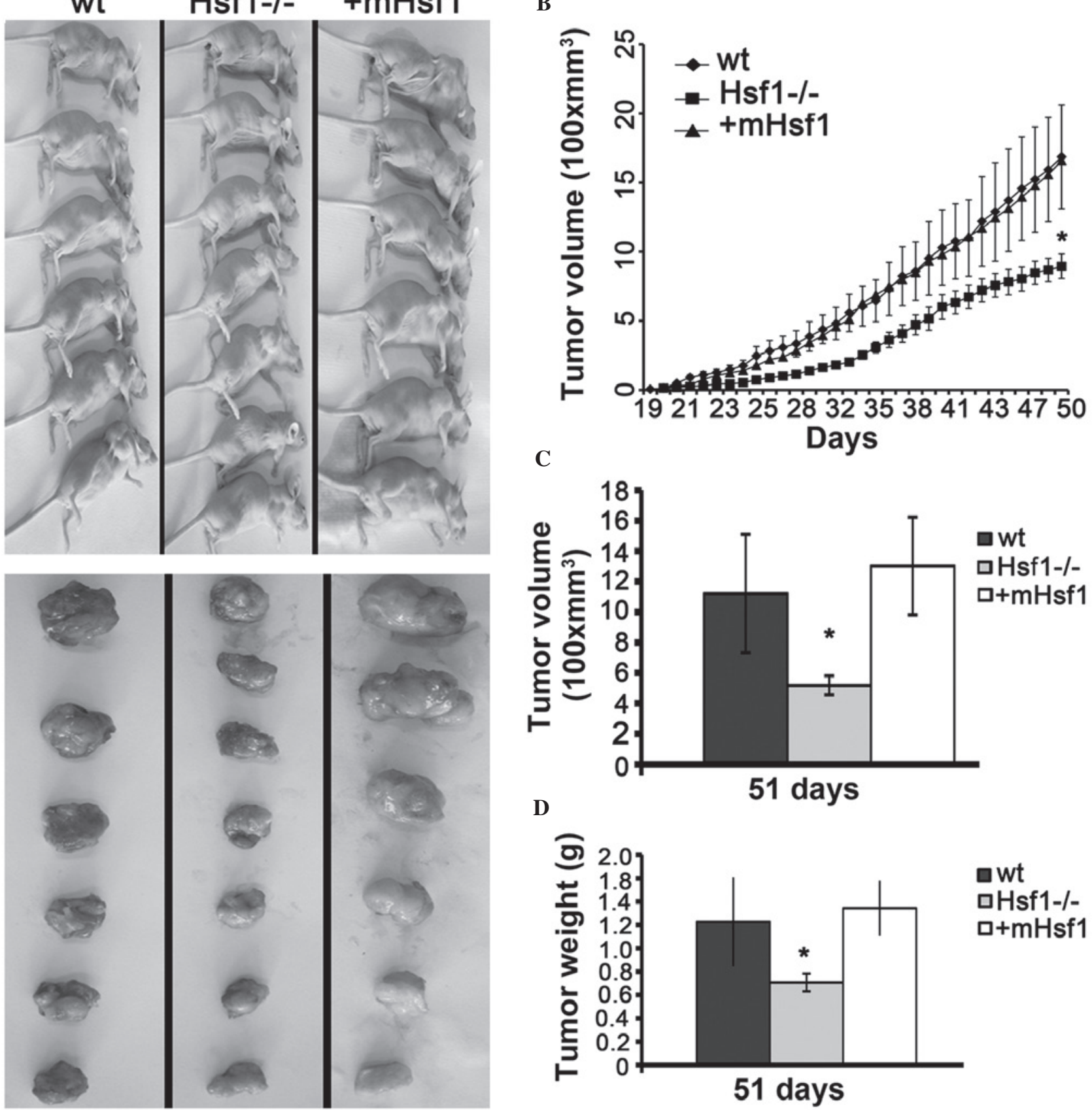

D
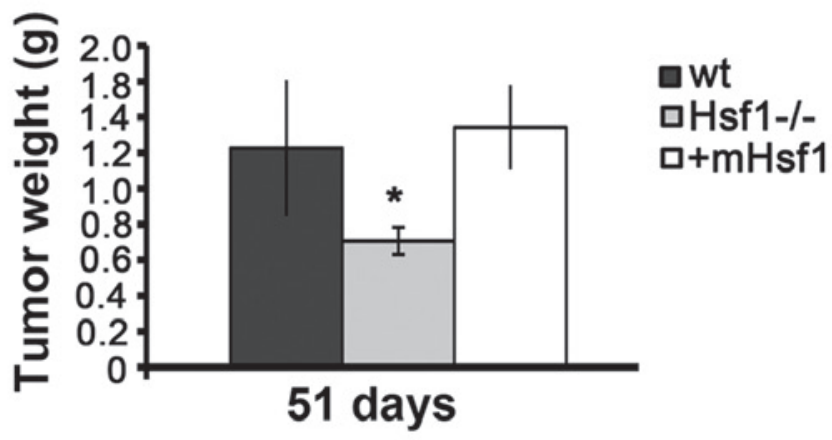

Figure 2. Knockout of Hsf1 inhibits the growth of MEF-derived fibroblastomas in athymic nude mice. (A) The athymic nude mice bearing engrafted tumors were sacrificed at day 50. The lower panel presents the solid tumors isolated from the athymic nude mice. (B) The growth of fibrosarcomas derived from the subcutaneously engrafted MEF cells in athymic nude mice. Tumor size was measured and calculated using the formula a(b) $2 / 2$, where a is the longest diameter of the tumor and $\mathrm{b}$ is the shortest diameter. (C) The weight of xenografted tumors in athymic nude mice. (D) The volume of fibrosarcomas in athymic nude mice. One-way analysis of variance was used. "P<0.05, Hsf1-/- group vs. MEF/wt and MEF/mHsf1 groups. Hsf1, heat shock factor 1; MEF, mouse embryonic fibroblast; wt, wild type; mHsf1, Hsf1 null MEF cells expressing mouse Hsf1.

is expressed in $\mathrm{MEF} / \mathrm{wt}$ and $\mathrm{MEF} / \mathrm{mHsf} 1$ cells, however not in $\mathrm{MEF} / \mathrm{Hsf} 1-/-$ cells. The proliferation of these three cell lines was investigated using an MTT and a colony formation assay. $\mathrm{MEF} / \mathrm{wt}$ and $\mathrm{MEF} / \mathrm{mHsf} 1$ cells exhibited a similar rate of proliferation and colony formation, and were observed to proliferate significantly faster than the MEF/Hsf1-/- cells (Fig. 1B-D). The result of the cell cycle analysis indicated that the number of $\mathrm{MEF} / \mathrm{wt}$ and $\mathrm{MEF} / \mathrm{mHsf} 1$ cells at $\mathrm{G}_{1}$ phase were 25 and $22 \%$ respectively, which was significantly increased to $45 \%$ in the MEF/Hsf1-/- cells $(\mathrm{P}<0.01$; Fig. 1E). By contrast, the number of MEF/Hsf1-/- cells at the $S$ and $G_{2}$ phases was significantly reduced compared with the $\mathrm{MEF} / \mathrm{wt}$ and $\mathrm{MEF} / \mathrm{mHsf} 1$ cells $(\mathrm{P}<0.05$; Fig. 1E). These results indicate that knockout of Hsf1 inhibits the proliferation of SV40/TAG transformed MEF cells in vitro by blocking the cell cycle at the $\mathrm{G}_{1}$ phase.

Knockout of Hsf1 inhibits the growth of fibroblastomas derived from MEF cell lines in athymic nude mice. SV40/TAG is able to completely transform cells into malignant tumor cells (16). 
A

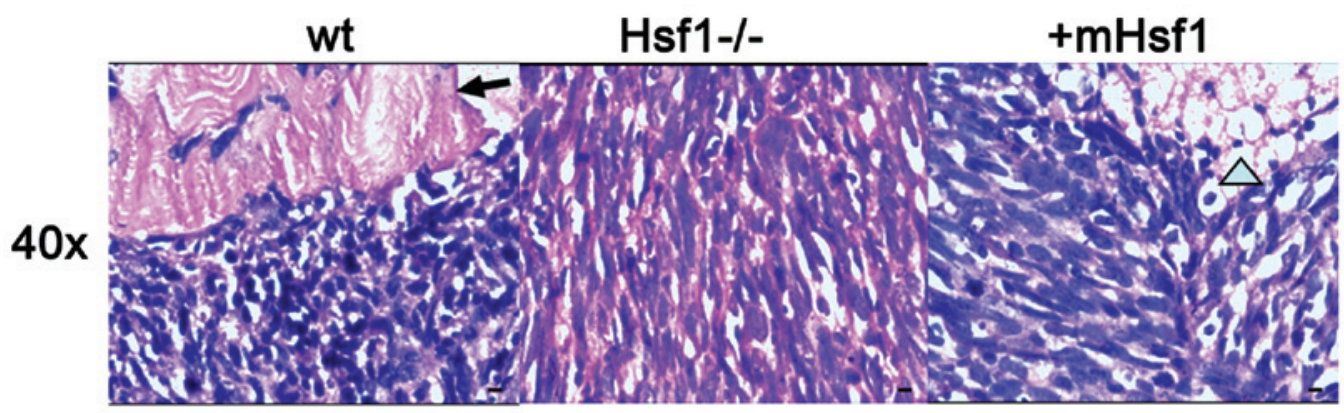

B

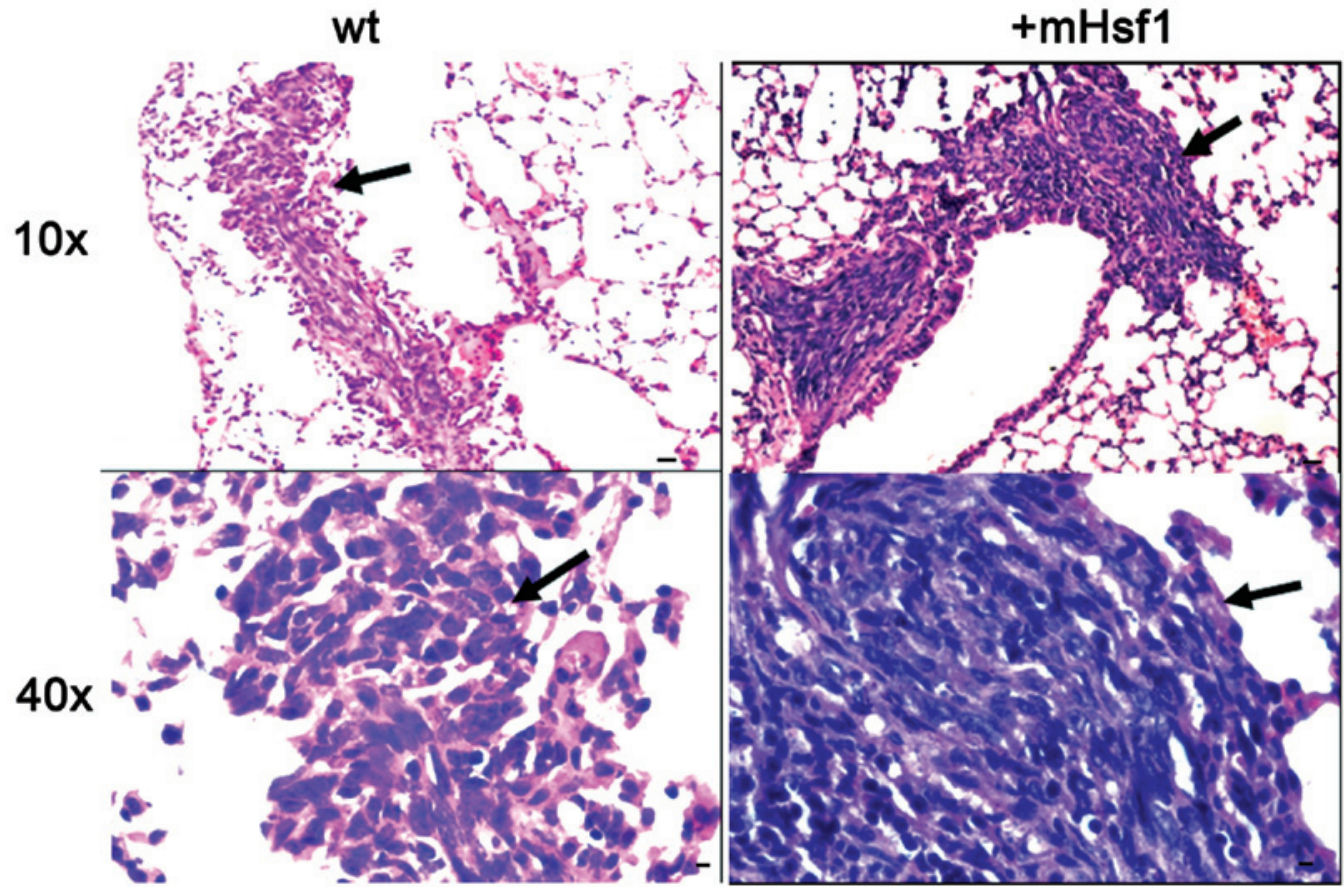

Figure 3. Histological analysis of the fibrosarcoma tissues in the athymic nude mice. (A) Images of H\&E stained tumor tissues as visualized through light microscopy. Arrow represents muscle infiltration; arrowhead indicates the fat infiltration. (B) Images of the H\&E stained lung metastatic tumor tissue from wt and mHsf1 fibrosarcoma bearing athymic nude mice. The arrow indicates metastatic tumor tissues. H\&E, hematoxylin and eosin; wt, wild type; Hsf1, heat shock factor 1; mHsf1, Hsf1 null MEF cells expressing mouse Hsf1.

To determine the roles of Hsf1 in the SV40/TAG-mediated malignant transformation of MEF cells, MEF/wt, MEF/Hsf1-/and $\mathrm{MEF} / \mathrm{mH}$ sf 1 cells were engrafted subcutaneously into athymic nude mice and the tumor formation and growth were measured. As presented in Fig. 2A, tumors had formed in all athymic nude mice by day 29 following engraftment. There was no significant difference in the time taken for tumor formation between the mice engrafted with $\mathrm{MEF} / \mathrm{wt}, \mathrm{MEF} / \mathrm{Hsf1-/-}$ and $\mathrm{MEF} / \mathrm{mHsf} 1$ cells. However, the growth of the MEF/wt and $\mathrm{MEF} / \mathrm{mHsf} 1$ tumors was significantly faster compared with that of the MEF/Hsf1-/- tumors ( $\mathrm{P}<0.05$; Fig. 2B). No difference in tumor growth rate was observed between the $\mathrm{MEF} / \mathrm{wt}$ and MEF/mHsf1 groups (Fig. 2A and B). Quantitative results indicated that the weights and volumes of the MEF/wt and $\mathrm{MEF} / \mathrm{mHs} 1$ tumors were significantly greater compared with

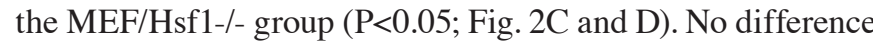
in tumor size and weight was observed between $\mathrm{MEF} /$ wt and $\mathrm{MEF} / \mathrm{mHsf} 1$ tumors. Histological studies confirmed that fibrosarcomas formed from all of the three cell lines engrafted into athymic nude mice (Fig. 3A). The MEF/wt and MEF/mHsf1 fibrosarcomas exhibited greater numbers of necrotic foci, and increased levels of pathological mitosis and peripheral muscle and fat infiltration compared with that of the MEF/Hsf1-/fibrosarcoma tissue. These data suggest that Hsf1 is involved in the regulation of tumor cell proliferation and development rather than tumor initiation in the SV40/TAG transformation model.

Knockout of Hsfl results in downregulation of tumor angiogenesis. In order to determine the metastatic potential of these fibrosarcomas, the main organs (brain, liver, lung, spleen and lymph nodes) of the tumor-bearing mice were screened using hematoxylin and eosin staining. Histology from 2 of $6 \mathrm{MEF} / \mathrm{wt}$ mice $(33.33 \%)$ and 1 of $6 \mathrm{MEF} / \mathrm{mHsf} 1$ mice (16.67\%) exhibited lung metastasis (Fig. 3B). No metastasis was observed in the $6 \mathrm{MEF} / \mathrm{Hsf} 1-/-$ mice. Further studies using immunohistochemical staining indicated that the expression of VEGF, CD34 and FVIII/Rag, the three hallmarks of angiogenesis, were significantly upregulated in the $\mathrm{MEF} / \mathrm{wt}$ and $\mathrm{MEF} / \mathrm{mHsf} 1$-derived fibrosarcoma tissue compared with the MEF/Hsf1-/- fibrosarcoma tissues (Fig. 4A and B). These data are consistent with previous reports (7) and suggest that 
A
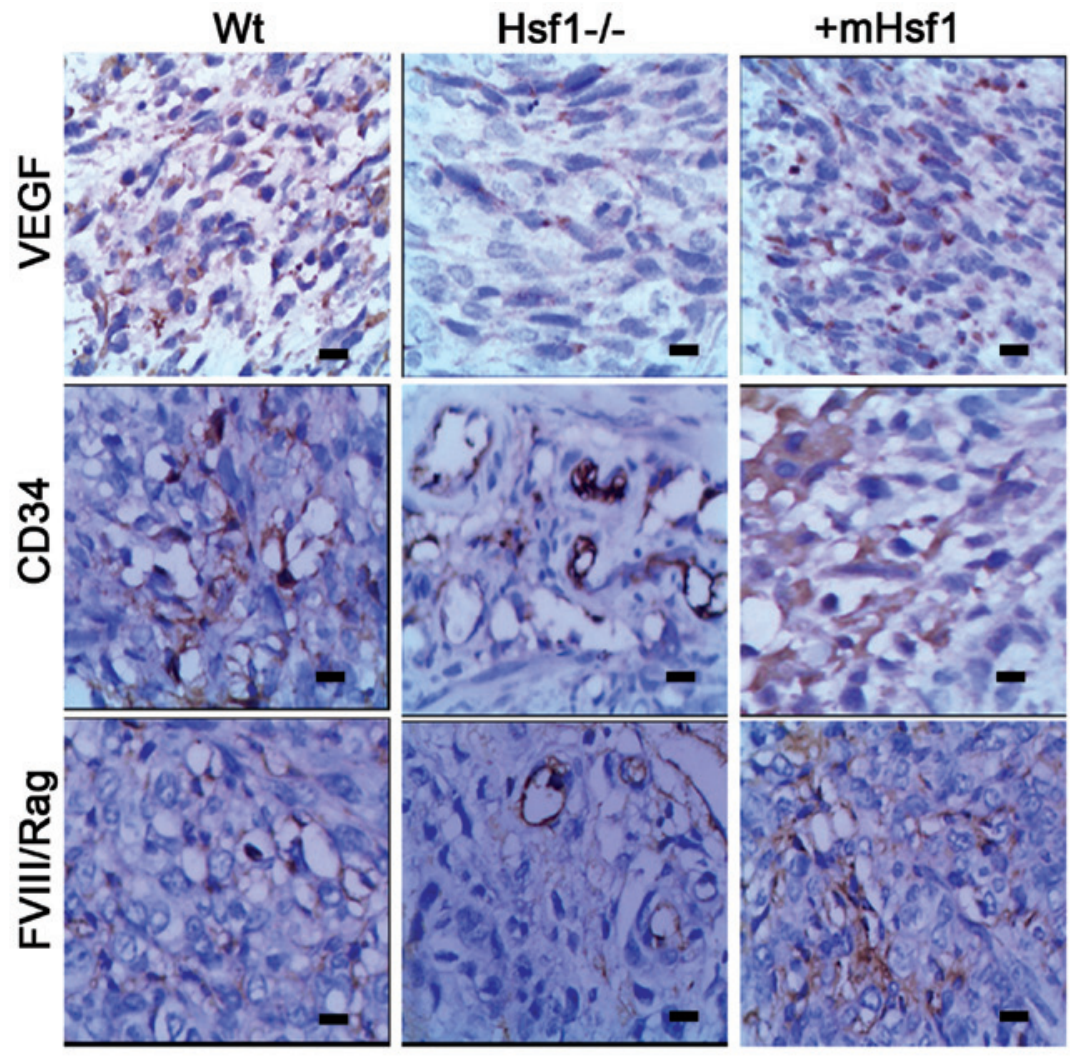

B

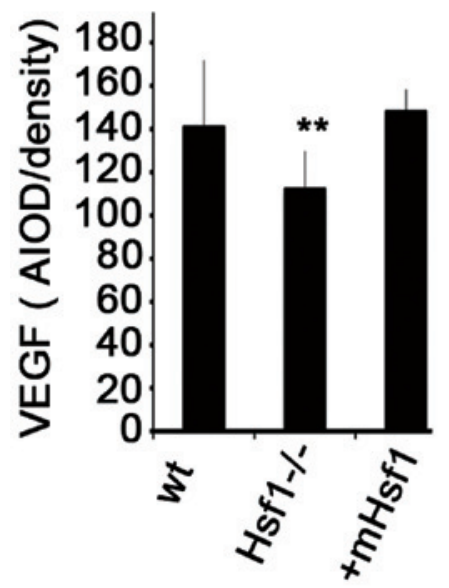

C

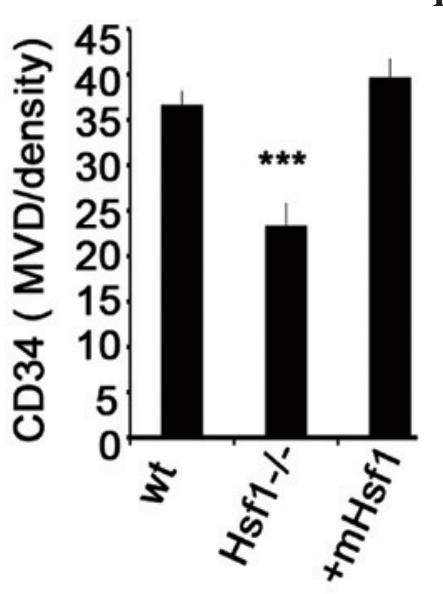

D

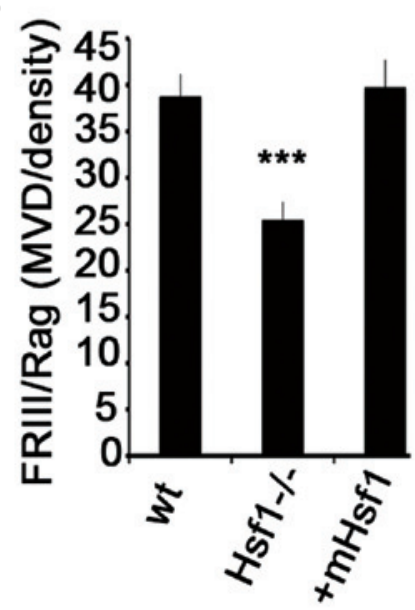

Figure 4. The expression of VEGF, CD34 and FVIII/Rag proteins in fibrosarcoma. (A) The immunohistochemical staining of the expression of VEGF, CD34 and FVIII/Rag proteins in the wt, Hsf1-/- and mHsf1 fibrosarcoma tissues. The images were taken using a 40X objective. (B) Quantification of the expression of VEGF in the fibrosarcoma tissue with the AIOD method. The quantification of the expression of (C) CD34 and (D) FVIII/Rag in the Hsf1-/- fibrosarcoma using the MVD method. Data are presented as the mean \pm standard deviation, one-way analysis of variance. ${ }^{* *} \mathrm{P}<0.01,{ }^{* * * *} \mathrm{P}<0.001 \mathrm{vs}$. wt and $\mathrm{mHsf} 1 \mathrm{cells}$. VEGF, vascular endothelial growth factor; CD34, cluster of differentiation 34; FVIII/Rag, factor VIII related antigen; wt, wild type; Hsf1, heat shock factor 1; mHsf1, Hsf1 null MEF cells expressing mouse Hsf1. AIOD, average integral optical density.

Hsf1 is involved in the regulation of tumor metastasis in SV40/TAG-induced fibrosarcoma.

Hsfl is associated with the expression of 53 and pRb proteins. Cell transformation by SV40/TAG is associated with p53 and pRb, two tumor suppressors. SV40/TAG binds to p53 and pRb and suppresses their transcriptional activities, which in turn are able to induce cell transformation (12). Hsf1 is reported to promote $\mathrm{p} 53$ protein degradation by upregulating the expression of the proteasome subunits proteasome subunit $\beta$ type- 5 and gankynin or $\alpha \mathrm{B}$-crystallin $(8,11)$. Therefore the deregulation of p53 or pRb expression may be involved in the growth inhibition of SV40/TAG transformed MEF/Hsf1-/- cells and the corresponding fibrosarcoma. Immunoblotting indicated that the protein expression levels of p53, pRb and their downstream target p21 were significantly upregulated in MEF/Hsf1-/- cells (Fig. 5A; lane 2) and the corresponding fibrosarcoma tissue (Fig. 5B; lanes 2, 5 and 8) when compared with the MEF/wt and $\mathrm{MEF} / \mathrm{mHsf} 1$ cells (Fig. 5A; lanes 1 and 3) and their corresponding fibrosarcoma tissues (Fig. 5B; lanes 1, 3, 4, 6, 7 and 9; and Fig. 5D). Furthermore, the expression of the SV40/TAG protein, which is similar to $\mathrm{p} 53$ and $\mathrm{pRb}$, was upregulated in 
A

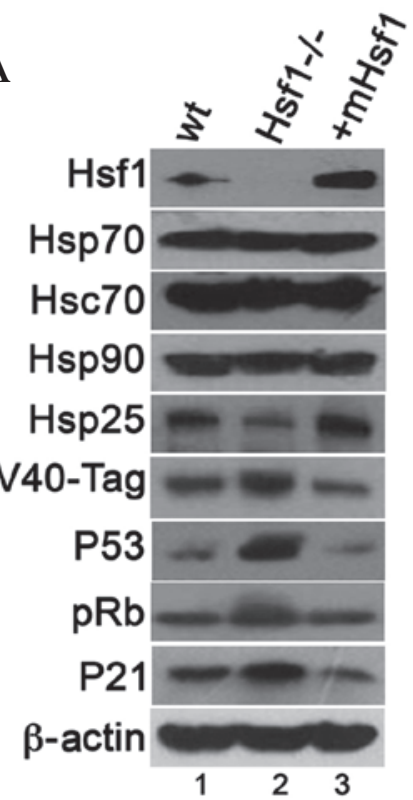

C

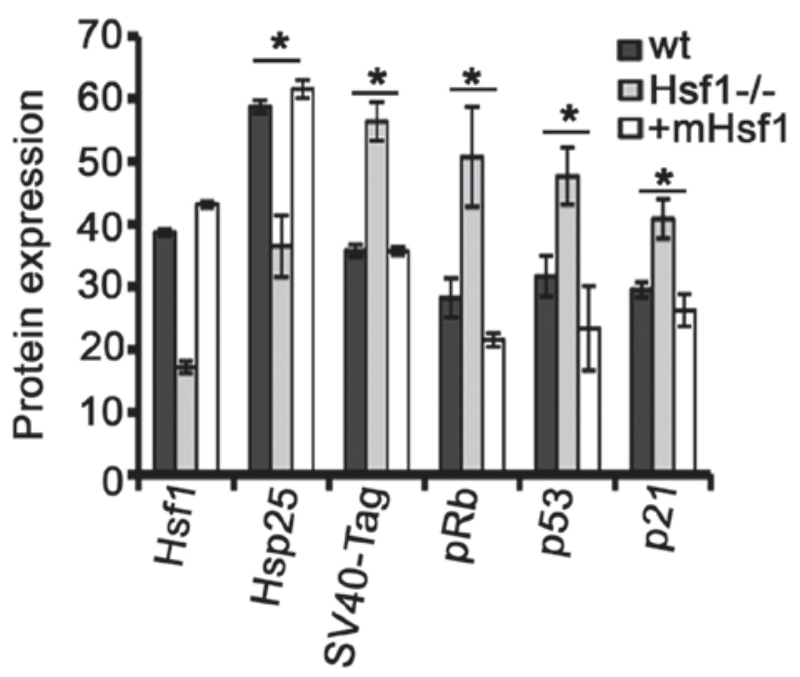

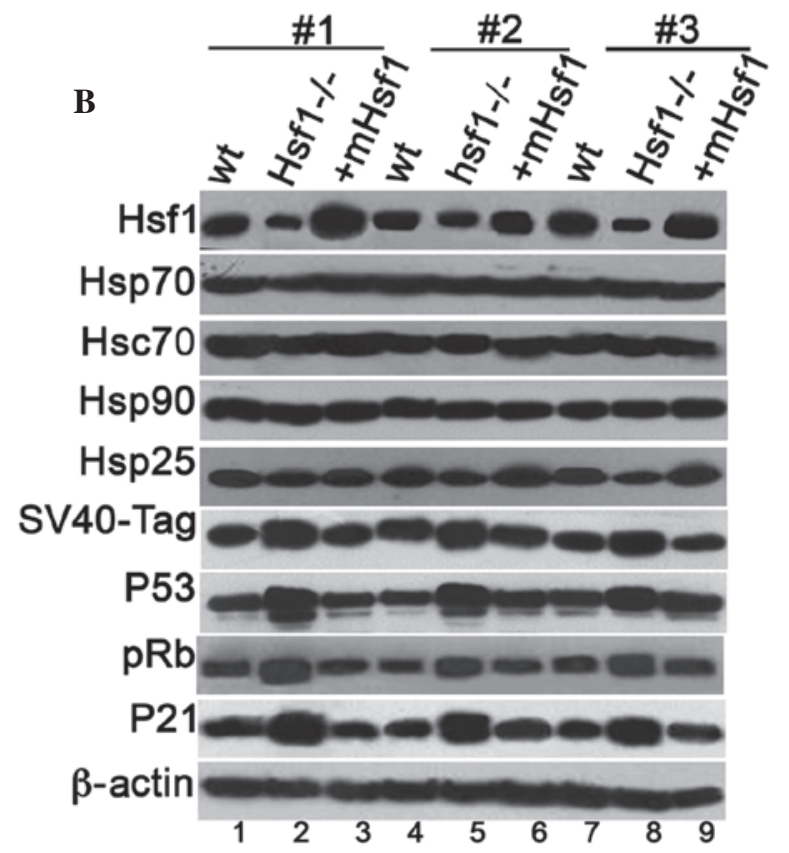

D

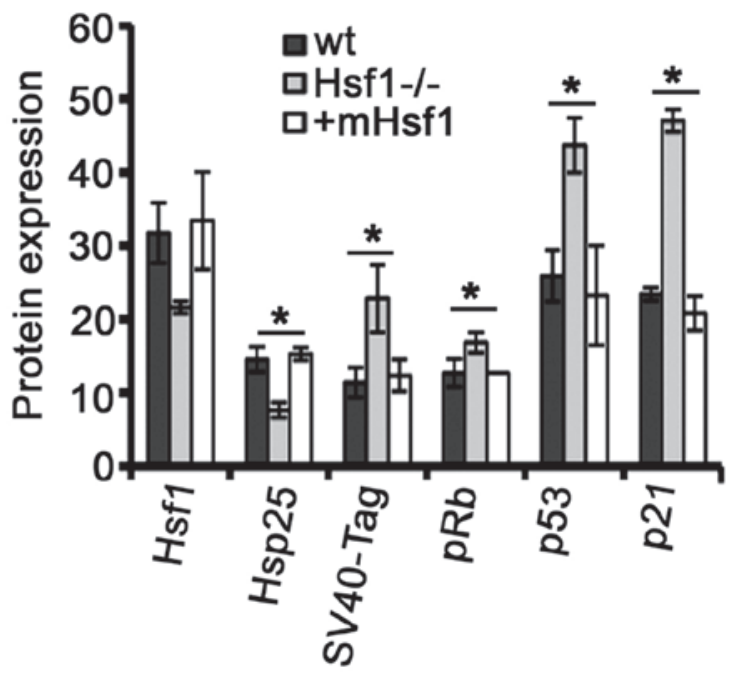

Figure 5. Hsf1 is associated with the expression of p53 and pRb proteins. (A) The expression of Hsf1, Hsp25, Hsc70, Hsp70, Hsp90, SV40/TAG, p53, p21 and $\mathrm{pRb}$ proteins were immunoblotted in MEF/wt (lane 1), MEF/hsf1-/- (lane 2) and MEF/mHsf1 (lane 3) cells. (B) The immunoblotting of the above proteins in the corresponding fibrosarcoma tissues. Experiments were repeated 3 times (\#1-3). The quantification of the expression of Hsf1, Hsp25, SV40/TAG, p53, pRB and p21 in the three (C) MEF cell lines and their corresponding (D) fibrosarcomas. ( $\mathrm{P}<0.05)$. hsf1, heat shock factor 1; pRb, phosphorylated retinoblastoma protein; Hsp25, heat shock protein 25; Hsc70, heat shock cognate protein 70; SV40/TAG, simian virus 40/T antigen; MEF, mouse embryonic fibroblast; wt, wild type; mHsf1, Hsf1 null MEF cells expressing mouse Hsf1.

fibrosarcoma/Hsf1-/- tissue compared with its expression in the fibrosarcomas derived from $\mathrm{MEF} / \mathrm{wt}$ and $\mathrm{MEF} / \mathrm{mHsf} 1$ cells (Fig. 5A and B). To investigate the expression of heat shock proteins underlying Hsf 1 in these tumor tissues, the expression levels of Hsp25, Hsp70 and Hsp90 were measured. The results indicated that the expression of Hsp25 was downregulated in $\mathrm{MEF} / \mathrm{Hsf1-/-}$ cells (Fig. 5A; lane 2) and in the corresponding fibrosarcoma (Fig. 5B; lanes 2, 5 and 8) when compared with the $\mathrm{MEF} /$ wt and $\mathrm{MEF} / \mathrm{mH}$ sf1 cells (Fig. 5A; lanes 1 and 3) and their corresponding fibrosarcomas (Fig. 5B). No difference in the expression levels of Hsp70, heat shock cognate protein 70 (Hsc70) and Hsp90 was observed in the three cell lines and their corresponding fibrosarcomas (Fig. 5A and B). Immunoblotting of $\beta$-actin indicated that protein loading was equal. Fig. 5C and D represent the quantification of the expression of the corresponding proteins in Fig. 5A and B. Taken together, these data indicate that knockout of Hsf1 results in the upregulation of p53 and additionally in the upregulation of pRb, p21 and SV40/TAG proteins. Upregulation of SV40/TAG does not inhibit p53 transcriptional activity in MEF/Hsf1-/and the derived fibrosarcoma tissues.

Knockout of Hsfl reduces the interaction between SV40/TAG and $p 53$ and $p R b$. The immunoblotting data indicates that knockout of Hsf1 inhibits the suppression of p53 transcriptional activity by SV40/TAG, which suggests that Hsf1 is involved in the association between p53 and SV40/TAG. To investigate this, the interaction between SV40/TAG and 
A

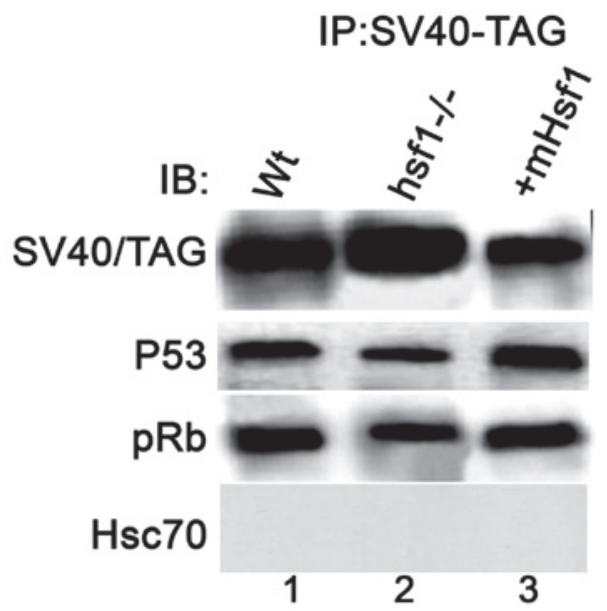

B

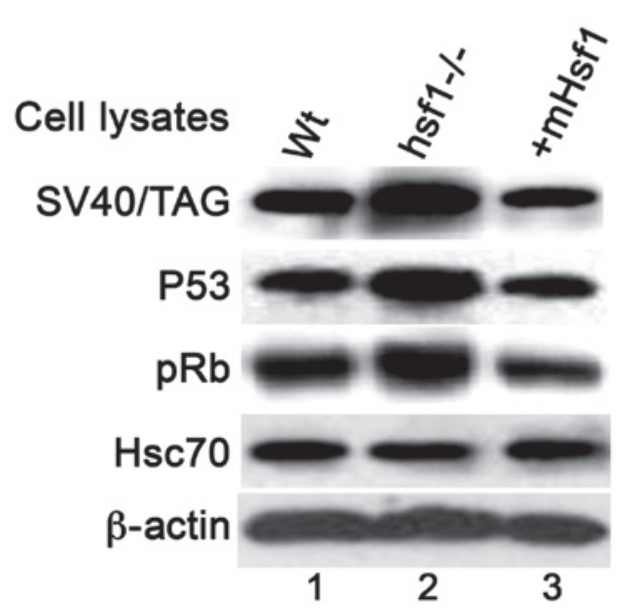

Figure 6. The interaction of SV40/TAG with $\mathrm{p} 53$, pRb and Hsc70 in the three MEF cell lines. (A) Coimmunoprecipitation of p53, pRb and Hsc70 with SV40/TAG. SV40/TAG proteins were immunoprecipitated from the cell lysates of the MEF/wt (lane 1), MEF/Hsf1-/- (lane 2) and MEF/mHsf1 (lane 3) cells. The immunoprecipitated protein complexes were immunoblotted with antibodies against p53,pRB and Hsc70. (B) The immunoblotting of the expression of SV40/TAG, p53, pRb and Hsc70 proteins in the cell lysates that were subjected to coimmunoprecipitation. SV40/TAG, simian virus 40/T antigen; $\mathrm{pRb}$, phosphorylated retinoblastoma protein; Hsc70, heat shock cognate protein 70; MEF, mouse embryonic fibroblast; wt, wild type Hsf1, heat shock factor 1; mHsf1, Hsf1 null MEF cells expressing mouse Hsf1; IP, immunoprecipitate; IB; immunoblot.

$\mathrm{p} 53 / \mathrm{pRb}$ was measured in the three types of MEF cells using immunoprecipitation assays. The amount of p53 and pRb proteins that were coimmunoprecipitated with SV40/TAG in the MEF/Hsf1-/- cells was reduced compared with that in the $\mathrm{MEF} / \mathrm{wt}$ and $\mathrm{MEF} / \mathrm{mHsf} 1$ cells. No interaction between SV40/TAG and Hsc70 was observed in the three MEF cell lines (data not shown). The expression of endogenous $\mathrm{p} 53, \mathrm{pRb}$, $\beta$-actin and ectopic SV40/TAG in the cell lysates that were applied for the immunoprecipitation assay are presented in Fig. 6B. These data indicate that the knockout of Hsf1 reduces the association of SV40/TAG with p53 and/or pRb.

\section{Discussion}

Hsf1 has been demonstrated to be associated with tumorigenesis in animal models and in humans (1). It has been reported that Hsf1 is important for the oncogenic processes mediated by Ras, p53 and E1A $(6,11)$. In the current study, evidence that Hsf1 is additionally involved in SV40/TAG-induced cell transformation is presented. $\mathrm{MEF} / \mathrm{wt}$ and $\mathrm{MEF} / \mathrm{Hsf1-/}$ cells were immortalized by SV40/TAG and formed fibrosarcomas following engraftment subcutaneously in athymic nude mice (Fig. 2). However, knockout of Hsf1 blocked the cell cycle at the $G_{1}$ phase and reduced the number of cells in the $\mathrm{S}$ and $\mathrm{G}_{2}$ phases. This resulted in the inhibition of $\mathrm{MEF} / \mathrm{Hsf1-/}$ cell proliferation in vitro and of $\mathrm{MEF} / \mathrm{Hsf1-/-fibrosarcoma}$ growth in vivo in the athymic nude mice (Figs. 1 and 2). Further investigation suggested that the knockout of $\mathrm{Hsf1}$ is able to upregulate the protein expression of the tumor suppressors p53, pRb and p21 in addition to SV40/TAG (Fig. 5). However, the associations between SV40/TAG and p53 and pRb were significantly reduced in MEF/Hsf 1-/- cells, which may provide an explanation for the slow growth of the Hsf1-/- fibrosarcoma. Furthermore, the data suggested that the knockout of Hsf1 suppressed lung metastasis of the fibrosarcoma by reducing angiogenesis (Fig. 3). Taken together, these data provide further evidence to support the fact that Hsf1 is an important factor for the growth and metastasis of viral oncogene-induced tumors.

There is substantial evidence in support of the involvement of Hsf 1 in tumor initiation and the development of tumors induced by p53-mutation, H-Ras and ErbB2-mutations in mouse models $(7,9)$. However, its roles in the tumorigenesis of viral oncogene induced tumors remain unclear. Jin et al (11) reported that MEF/wt and MEF/Hsf1-/- cells were immortalized by E1A, however the proliferation and colony formation of the E1A-immortalized MEF/Hsf1-/- cells was slower compared with the MEF/wt cells. Upregulation of p53 protein through the reduced expression of $\alpha \mathrm{B}$-crystallin expression in $\mathrm{MEF} / \mathrm{Hsf1-/}$ - cells was suggested to explain this slow proliferation (11). $\alpha \mathrm{B}$-crystallin has been reported to form a complex with F-box only protein 7 and p53, mediating p53 degradation through the proteasomal pathway (11). However, there are inadequate in vivo studies to suggest whether the E1A-immortalized MEF cells are malignant. In the current study, SV40/TAG transformed MEF/Hsf1-/- and MEF/wt cells in vitro, and the transformed cells formed fibrosarcomas when subcutaneously engrafted into athymic nude mice. However, the growth of the MEF/Hsf1-/- cells and their corresponding fibrosarcomas was significantly slower compared with $\mathrm{MEF} / \mathrm{wt}$ cells. These data indicate that Hsf1 is important in maintaining viral tumor cell proliferation rather than initiating transformation.

SV40/TAG induces cell transformation by targeting two tumor suppressors, p53 and pRb. SV40/TAG interacts with and inhibits the transcriptional activities of p53 and $\mathrm{pRb}$, which result in the deregulation of the cell cycle and cell transformation $(13,21)$. The results of the current study indicate that the expression of $\mathrm{p} 53$ and $\mathrm{pRb}$ are upregulated in $\mathrm{MEF} / \mathrm{Hsf1} 1-/$ - cells, which are consistent with the previously reported results, in which Hsf1 knockout resulted in p53 protein stabilization by E1A (11). Although it is unclear which mechanism results in the accumulation of p53 and $\mathrm{pRb}$ proteins in SV40/TAG-transformed MEF/Hsf1-/- cells, the current study indicated that in addition SV40/TAG protein was upregulated in the MEF/Hsf1-/- cells and fibrosarcoma, and the upregulation of SV40/TAG did not inhibit the transcriptional regulation of $\mathrm{p} 21$ expression by $\mathrm{p} 53$. This suggests 
that the association between SV40/TAG and p53 or pRb may be blocked in MEF/Hsf1-/- cells and fibrosarcoma tissue. The coimmunoprecipitation data demonstrated that the interaction between SV40/TAG and p53 or pRb is significantly reduced in $\mathrm{MEF} / \mathrm{Hsf1-/}$ - cells compared with that in $\mathrm{MEF} / \mathrm{wt}$ and $\mathrm{MEF} / \mathrm{mHsf} 1$ cells, despite the SV40/TAG-induced upregulation of p53 and pRb proteins in MEF/Hsf1-/- cells. It remains unclear which mechanism is responsible for the reduced interaction between SV40/TAG and p53 or pRb in the $\mathrm{MEF} / \mathrm{Hsf1-/-}$ cells. Xi et al (7) reported that knockdown of Hsf1 is able to reduce the interaction between Raf and Hsp90, which then in turn reduces the signal transduction from ErbB2 to the mitogen activated protein kinase pathway, and the ErbB2-mutation-induced breast cancer occurrence in mouse models. The current study measured the expression of additional heat shock proteins (Hsp90, Hsp70 and Hsp25) and observed that Hsp25 alone is significantly reduced in $\mathrm{MEF} / \mathrm{Hsf1-/}$ cells and the corresponding fibrosarcoma, suggesting that Hsp25 is the predominant target of Hsf1 in SV40/TAG-transformed MEF cells and their corresponding fibrosarcomas. A previous study reported that heat shock proteins are upregulated in SV40 transformed cells (22), suggesting that heat shock proteins in general serve a role in SV40/TAG transformation (e.g. anti-apoptosis and cell cycle proliferation). However, whether Hsp25 is specifically involved in regulating the association between SV40/TAG and p53 or $\mathrm{pRb}$ requires further investigation.

Invasion and metastasis are two important hallmarks of malignant tumors, and a number of factors have been demonstrated to be involved in these processes [e.g. VEGF, hypoxia-inducible factor $1 \alpha(\operatorname{Hif} 1 \alpha)$ and matrix metalloproteinases]. It has been reported that Hsf1 is involved in the regulation of tumor metastasis in breast cancer $(7,9)$. Upregulation of Hsf1 is associated with worsened prognosis in breast cancer, HCC and other types of tumor (9). Xi et al (7) reported that knockdown of Hsf1 inhibits breast cancer development and transforming growth factor- $\beta 1$ induced epithelial-mesenchymal transition. Gabai et al (23) reported that Hsf1 regulates breast cancer progression by regulating the expression of Hif $1 \alpha$ and RNA regulator Human antigen R. Mendillo et al (9) demonstrated that in addition to regulating heat shock proteins, Hsf1 regulates the expression of genes that regulate breast cancer cell proliferation, migration and invasion (9). In the current study, the fibrosarcomas derived from $\mathrm{MEF} / \mathrm{wt}$ and $\mathrm{MEF} / \mathrm{mHsf} 1$ cells were observed to metastasize to the lungs, however, the fibrosarcoma derived from $\mathrm{MEF} / \mathrm{Hsf1-/-} \mathrm{did} \mathrm{not.} \mathrm{Further} \mathrm{analysis} \mathrm{indicated} \mathrm{that} \mathrm{knockout}$ of Hsf1 reduced the angiogenesis of the fibrosarcoma. This is reflected by the low expression of the angiogenesis markers including VEGF, CD34 and FVIII/Rag in the MEF/Hsf1-/fibrosarcoma compared with the fibrosarcomas that were derived from MEF/wt and MEF/mHsf1 cells. Tumor analysis demonstrated that $\mathrm{MEF} / \mathrm{wt}$ and $\mathrm{MEF} / \mathrm{mHsf} 1$ fibrosarcomas grew faster, contained larger mitotic cells and more fat, and exhibited increased muscle invasion when compared with the MEF/Hsf1-/- fibrosarcoma. This suggests that Hsf1 is an important factor in regulating tumor malignancy and metastasis. However, the mechanism behind this regulation remains unclear. Hsp25 has been reported to be associated with cancer metastasis $(24,25)$ and has been targeted as a diagnostic marker for a number of tumors (26). In addition, Hsp25 was demonstrated to regulate the stability of a number of transcription factors such as GATA-1 and Snail (27,28), which are important in promoting tumor cell growth and metastasis. The current study indicates that Hsp25 is downregulated in the $\mathrm{MEF} / \mathrm{Hsf} 1-/-$ cells and fibrosarcoma tissue. However, whether the downregulation of Hsp25 is involved in regulating the dissociation of SV40-TAG from p53 and pRb, and the reduced

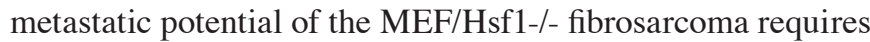
further investigation. Taken together, these data provide further evidence to support the fact that Hsf1 is an important regulator in tumor metastasis.

Using the SV40/TAG immortalized MEF cell model, the current study demonstrated that Hsf1 is involved in the regulation of viral-oncogene induced tumor growth and metastasis rather than tumor initiation. These data provide further evidence to suggest that Hsf1 may be a potential therapeutic target for viral-oncogene-induced tumors.

\section{Acknowledgements}

The current study was supported by the National Natural Science Foundation of China (grant nos. 30971508 and 81270985).

\section{References}

1. Calderwood SK: Elevated levels of HSF1 indicate a poor prognosis in breast cancer. Future Oncol 8: 399-401, 2012.

2. Jin X, Moskophidis D and Mivechi NF: Heat shock transcription factor 1 is a key determinant of HCC development by regulating hepatic steatosis and metabolic syndrome. Cell Metab 14: 91-103, 2011.

3. Santagata S, Hu R, Lin NU, Mendillo ML, Collins LC Hankinson SE, Schnitt SJ, Whitesell L, Tamimi RM, Lindquist S and Ince TA: High levels of nuclear heat-shock factor 1 (HSF1) are associated with poor prognosis in breast cancer. Proc Natl Acad Sci USA 108: 18378-18383, 2011.

4. Whitesell $\mathrm{L}$ and Lindquist $\mathrm{S}$ : Inhibiting the transcription factor HSF1 as an anticancer strategy. Expert Opin Ther Targets 13: 469-478, 2009.

5. Chen Y, Chen J, Loo A, Jaeger S, Bagdasarian L, Yu J, Chung F, Korn J, Ruddy D, Guo R, et al: Targeting HSF1 sensitizes cancer cells to HSP90 inhibition. Oncotarget 4: 816-829, 2013.

6. Dai C, Whitesell L, Rogers AB and Lindquist S: Heat shock factor 1 is a powerful multifaceted modifier of carcinogenesis. Cell 130: 1005-1018, 2007.

7. Xi C, Hu Y, Buckhaults P, Moskophidis D and Mivechi NF: Heat shock factor Hsf1 cooperates with ErbB2 (Her2/Neu) protein to promote mammary tumorigenesis and metastasis. J Biol Chem 287: 35646-35657, 2012.

8. Lecomte S, Desmots F, Le Masson F, Le Goff P, Michel D, Christians ES and Le Dréan Y: Roles of heat shock factor 1 and 2 in response to proteasome inhibition: Consequence on p53 stability. Oncogene 29: 4216-4224, 2010.

9. Mendillo ML, Santagata S, Koeva M, Bell GW, Hu R, Tamimi RM, Fraenkel E, Ince TA, Whitesell $\mathrm{L}$ and Lindquist $\mathrm{S}$ : HSF1 drives a transcriptional program distinct from heat shock to support highly malignant human cancers. Cell 150: 549-562, 2012.

10. Xu R, Zhang X, Zhang W, Fang Y, Zheng S and Yu XF: Association of human APOBEC3 cytidine deaminases with the generation of hepatitis virus $\mathrm{B} \times$ antigen mutants and hepatocellular carcinoma. Hepatology 46: 1810-1820, 2007.

11. Jin X, Moskophidis D, Hu Y, Phillips A and Mivechi NF: Heat shock factor 1 deficiency via its downstream target gene alphaB-crystallin (Hspb5) impairs p53 degradation. J Cell Biochem 107: 504-515, 2009.

12. Colvin EK, Weir C, Ikin RJ and Hudson AL: SV40 TAg mouse models of cancer. Semin Cell Dev Biol 27: 61-73, 2014. 
13. Ludlow JW, Shon J, Pipas JM, Livingston DM and DeCaprio JA: The retinoblastoma susceptibility gene product undergoes cell cycle-dependent dephosphorylation and binding to and release from SV40 large T. Cell 60: 387-396, 1990.

14. Pallas DC, Shahrik LK, Martin BL, Jaspers S, Miller TB, Brautigan DL and Roberts TM: Polyoma small and middle $\mathrm{T}$ antigens and SV40 small $t$ antigen form stable complexes with protein phosphatase 2A. Cell 60: 167-176, 1990.

15. Chen W, Possemato R, Campbell KT, Plattner CA, Pallas DC and Hahn WC: Identification of specific PP2A complexes involved in human cell transformation. Cancer Cell 5: 127-136, 2004.

16. Ahuja D, Sáenz-Robles MT and Pipas JM: SV40 large T antigen targets multiple cellular pathways to elicit cellular transformation. Oncogene 24: 7729-7745, 2005.

17. Yang $J$ and DeFranco DB: Differential roles of heat shock protein 70 in the in vitro nuclear import of glucocorticoid receptor and simian virus 40 large tumor antigen. Mol Cell Biol 14: 5088-5098, 1994

18. Wapnir IL, Wartenberg DE and Greco RS: Three dimensional staging of breast cancer. Breast Cancer Res Treat 41: 15-19, 1996

19. Kraan MC, Smith MD, Weedon H, Ahern MJ, Breedveld FC and Tak PP: Measurement of cytokine and adhesion molecule expression in synovial tissue by digital image analysis. Ann Rheum Dis 60: 296-298, 2001.

20. Hu YZ, Zhang J, Li S, Wang C, Chu L, Zhang Z, Ma Z, Wang M, Jiang Q, Liu G, et al: The transcription activity of heat shock factor $4 \mathrm{~b}$ is regulated by FGF2. Int J Biochem Cell Biol 45: 317-325, 2013

21. Sáenz Robles MT and Pipas JM: T antigen transgenic mouse models. Semin Cancer Biol 19: 229-235, 2009.
22. Omar RA and Lanks KW: Heat shock protein synthesis and cell survival in clones of normal and simian virus 40-transformed mouse embryo cells. Cancer Res 44: 3976-3982, 1984.

23. Gabai VL, Meng L, Kim G, Mills TA, Benjamin IJ and Sherman MY: Heat shock transcription factor Hsf1 is involved in tumor progression via regulation of hypoxia-inducible factor 1 and RNA-binding protein HuR. Mol Cell Biol 32: 929-940, 2012

24. Shiota M, Bishop JL, Nip KM, Zardan A, Takeuchi A, Cordonnier T, Beraldi E, Bazov J, Fazli L, Chi K, et al: Hsp27 regulates epithelial mesenchymal transition, metastasis, and circulating tumor cells in prostate cancer. Cancer Res 73: 3109-3119, 2013

25. Pavan S, Musiani D, Torchiaro E, Migliardi G, Gai M, Di Cunto F, Erriquez J, Olivero M and Di Renzo MF: HSP27 is required for invasion and metastasis triggered by hepatocyte growth factor. Int J Cancer 134: 1289-1299, 2014.

26. Mese H, Sasaki A, Nakayama S, Yoshioka N, Yoshihama Y, Kishimoto K and Matsumura T: Prognostic significance of heat shock protein 27 (HSP27) in patients with oral squamous cell carcinoma. Oncol Rep 9: 341-344, 2002.

27. Wettstein G, Bellaye PS, Kolb M, Hammann A, Crestani B, Soler P, Marchal-Somme J, Hazoume A, Gauldie J, Gunther A, et al: Inhibition of HSP27 blocks fibrosis development and EMT features by promoting Snail degradation. FASEB J 27: 1549-1560, 2013.

28. de Thonel A, Vandekerckhove J, Lanneau D, Selvakumar S, Courtois G, Hazoume A, Brunet M, Maurel S, Hammann A, Ribeil JA, et al: HSP27 controls GATA-1 protein level during erythroid cell differentiation. Blood 116: 85-96, 2010. 